Pacific

Journal of

Mathematics

SHARP SIZE ESTIMATES FOR CAPILLARY FREE SURFACES WITHOUT GRAVITY

$$
\text { XI-NAN MA }
$$




\title{
SHARP SIZE ESTIMATES FOR CAPILLARY FREE SURFACES WITHOUT GRAVITY
}

\author{
XI-NAN MA
}

\begin{abstract}
For the equation of constant mean curvature with prescribed constant contact angle boundary condition, using the unique continuation of analytic function, we get a minimum principle for a combination of the solution and its gradient. Thus we get the endpoint case for $P$-function (Sperb, 1981) and in fact answer an open question which appeared twenty years ago in Payne \& Philippin, 1977, 1979 and Sperb, 1981. As an application, sharp size and shape estimetes for capillary free surface without gravity are obtained.
\end{abstract}

\section{Introduction and Results.}

The capillary surface of a liquid contained in a vertical tube with arbitrary cross section $\Omega$ in the outer space has the shape of surface of constant mean curvature with constant contact angle $\theta_{o}$ against the wall of the tube. Let the capillary surface be expressed non-parametrically as the graph of a function $u$ defined over the cross section $\Omega$. How does the boundary geometry of $\Omega$ and the contact angle $\theta_{o}$ influence the size and shape of the capillary free surface?

For the convexity of the capillary free surface, in [2], Chen and Huang have shown if $\Omega$ is a bounded convex domain in the plane and $\theta_{o}=0$, then the corresponding capillary surface is also convex. Finn [3] provided an example to show if $\theta_{o} \neq 0$ the result is in general false.

In $[\mathbf{1}, \mathbf{1 0}]$, Chen and Sakaguchi showed if $\Omega$ be a bounded smooth convex domain in $R^{2}, 0<\theta_{o}<\frac{\pi}{2}$, the capillary free surface over $\Omega$ has only one minimal point. From the convexity of the surface as $\theta_{o}=0$, we know for any $\theta_{o}\left(0 \leq \theta_{o}<\frac{\pi}{2}\right)$, the minimal point is unique.

In this paper we consider the influence of boundary geometry and the contact angle $\theta_{o}\left(0 \leq \theta_{o}<\frac{\pi}{2}\right)$ on the size and shape for the capillary free surface without gravity. Precisely, let $\Omega$ be a bounded convex domain in $R^{2}$ with smooth boundary $\partial \Omega$. Give a positive constant $H$, consider the following equations:

$$
\sum_{i=1}^{2} D_{i}\left(\frac{u_{i}}{\sqrt{1+|D u|^{2}}}\right)=2 H \quad \text { in } \quad \Omega
$$




$$
\frac{u_{n}}{\sqrt{1+|D u|^{2}}}=\cos \theta_{o} \quad \text { on } \quad \partial \Omega .
$$

Where $u_{i}, i=1,2$ are partial derivatives of $u, n$ denotes the unit outer normal to $\partial \Omega, u_{n}$ denotes the direction derivative of $u$ along $n$, and $\theta_{o}$ $\left(0 \leq \theta_{o}<\frac{\pi}{2}\right)$ is the constant with $2 H|\Omega|=\cos \theta_{o}|\partial \Omega|(|\Omega|$ is the area of $\Omega$ and $|\partial \Omega|$ is the length of $\partial \Omega$ ). The graph of solution $u$ to (1.1)-(1.2) described a capillary free surface without gravity over the cross section $\Omega$.

Let $A \in \partial \Omega$ be a point corresponding to a minimum boundary value of $u, B \in \partial \Omega$ be a point corresponding to a maximum boundary value of $u$, $C \in \Omega$ be the unique minimal (critical) point of $u$ and $k(x)$ be the curvature of $\partial \Omega$ at $x \in \partial \Omega$. Now we state our theorems:

Theorem 1. Let $u \in C^{3}(\bar{\Omega})$ be a solution to (1.1)-(1.2), then the following inequalities hold

1):

$$
\begin{aligned}
u(A)-u(C) & \leq \frac{1-\sin \theta_{o}}{H} \\
k(A) & \leq \frac{H}{\cos \theta_{o}},
\end{aligned}
$$

2):

$$
\begin{aligned}
u(B)-u(C) & \geq \frac{1-\sin \theta_{o}}{H} \\
k(B) & \geq \frac{H}{\cos \theta_{o}} .
\end{aligned}
$$

If one of the equality signs of (1.3)-(1.6) holds then $\Omega$ is a disk of radius $\frac{\cos \theta_{o}}{H}$ and

$$
\begin{aligned}
& u(x)-u(C) \equiv \frac{1-\sin \theta_{o}}{H} \quad \text { on } \quad \partial \Omega \\
& k(x) \equiv \frac{H}{\cos \theta_{o}} \quad \text { on } \quad \partial \Omega .
\end{aligned}
$$

Conversely, (1.7)-(1.8) holds on $\partial \Omega$ if $\Omega$ is a disk of radius $\frac{\cos \theta_{o}}{H}$.

The proof of Theorem 1 is based on Hopf maximum principle [9] and the following minimum principle.

Theorem 2. Let $u \in C^{3}(\bar{\Omega})$ be a solution to (1.1)-(1.2), then the function

$$
P(x)=2-2\left(1+|D u|^{2}\right)^{-\frac{1}{2}}-2 H u
$$

attains its minimum on the boundary $\partial \Omega$, unless $P(x)$ is a constant on $\bar{\Omega}$. 
In [7], Payne and Philippin had proved a similar maximum principle for the above function $P(x)$ that under the same condition it also attains its maximum on $\partial \Omega$ unless $P(x)$ is a constant in $\Omega$.

Since our theorems concern only qualitative property of the solution, so only under the hypothesis of the existence of the solution we prove theorems. For the existence of solution and background details we refer the reader to the sources [4].

According to the work Nirenberg [5] or [4] we conclude that $u$ is an analytic function in $\Omega$, a feature which will be used in this paper.

In Section 2 we will give the the proof of Theorem 2 which is based on the unique continuation of analytic function. Section 3 contains a proof of Theorem 1 and a Corollary, which give the estimates of capillary free surface area using the volume of a liquid, $|\Omega|, \theta_{o}, u(A)$ and $u(B)$.

We conclude the introduction with some notations and an identity for Equation (1.1). Let $\Omega$ be a bounded convex smooth domain in the plane. We introduce curvilinear coordinate system $(r, s)$, where $s$ represents arc length along $\partial \Omega$ and $r\left(x_{1}, x_{2}\right)$ is the distance from a point $x=\left(x_{1}, x_{2}\right)$ in $\Omega$ to $\partial \Omega$. As in [11], we denotes $n=\left(n^{1}, n^{2}\right)$ the unit outward normal to $\partial \Omega$, $T=\left(T^{1}, T^{2}\right)$ is the unit tangent vector of $\partial \Omega$. The summation convention over repeated indices (from 1 to 2 ) will be employed. Assume that a function $u\left(x_{1}, x_{2}\right)$ is smooth in $\bar{\Omega}$, the following abbreviations will be adopted

$$
u_{1}=\frac{\partial u}{\partial x_{1}}, \quad u_{2}=\frac{\partial u}{\partial x_{2}}, \quad u_{i j}=\frac{\partial^{2} u}{\partial x_{i} \partial x_{j}}, \ldots, \quad v=1+|D u|^{2} .
$$

Following [11], we define the normal derivative $\frac{\partial u}{\partial n}$ of $u$ by

$$
u_{n}=\lim _{r \rightarrow 0} \frac{1}{r}(u(x)-u(x-r n))=u_{i} n^{i} .
$$

On $\partial \Omega$ we can also define a tagential derivative $\frac{\partial u}{\partial s}$ of $u$ by

$$
u_{s}=u_{i} T^{i}
$$

Then we have the following formulas on $\partial \Omega$

$$
\begin{aligned}
& u_{s s}=\frac{\partial^{2} u}{\partial s^{2}}=u_{i j} T^{i} T^{j}-k u_{i} n^{i} \\
& u_{n n}=\frac{\partial^{2} u}{\partial n^{2}}=u_{i j} n^{i} n^{j} \\
& u_{n s}=\frac{\partial}{\partial s}\left(\frac{\partial u}{\partial n}\right)=u_{i j} n^{i} T^{j}+k u_{s} \\
& u_{s n}=u_{n s}-k u_{s} .
\end{aligned}
$$

Using curvilinear coordinate system, Equation (1.1) implies the following formula on $\partial \Omega$

$$
u_{n n}\left(1+u_{s}^{2}\right)=2 H v^{\frac{3}{2}}-\left(u_{s s}+k u_{n}\right) v+\left(u_{s s} u_{s}^{2}+2 u_{s} u_{n} u_{n s}-k u_{n} u_{s}^{2}\right)
$$


which will be used in Section 3 to prove Theorem 1 .

Remark. The formula (1.4) is implicit contained in [7].

\section{A minimum principle.}

We consider the boundary value problem (1.1)-(1.2) in a strict convex bounded domain $\Omega$ in $R^{2}$ with smooth boundary $\partial \Omega$, and define the following function:

$$
P^{\alpha}(x)=2-2\left(1+|D u|^{2}\right)^{-\frac{1}{2}}-2 \alpha H u .
$$

We know that $P^{\alpha}(x)$ takes its maximum value at the critical point for $\alpha \geq 2$ [6], and on the boundary $\partial \Omega$ for $\alpha \leq 1$ [7]. We concentrate now our attention on $\alpha \in[1,2]$, and state the following:

Lemma $2.1([7,8])$. The function $P^{\alpha}(x)$ defined in (2.1) satisfies the following elliptic differential equation:

$$
\begin{aligned}
& \left(\delta_{i j}-u_{i} u_{j} v^{-1}\right) P_{i j}^{\alpha}-\left[2 H v^{-\frac{1}{2}} u_{i}+2 v^{-1}|D u|^{-2} u_{k} u_{k i}\right. \\
& \left.+2(\alpha-2) H v^{-\frac{1}{2}}|D u|^{-2} u_{i}-2 v^{-2}|D u|^{-2} u_{k} u_{l} u_{k l} u_{i}\right] P_{i}^{\alpha} \\
& =4(\alpha-1)(\alpha-2) H^{2} v^{-\frac{1}{2}}
\end{aligned}
$$

where $\delta_{i j}$ is Kronecker symbol.

For the proof of Lemma 2.1, we make use of Definition (2.1) and of the following identity (valid in $R^{2}$ only):

$$
u_{i j} u_{i} u_{j}|D u|^{2} \equiv|D u|^{2}(\Delta u)^{2}+2 u_{i} u_{i j} u_{k} u_{k j}-2 \Delta u u_{i} u_{j} u_{i j} .
$$

The details of the computations are omitted here since they had been given in $[7]$.

From Lemma 2.1 and Hopf maximum principle [9] we conclude that $P^{\alpha}(x)$ takes its minimum value either on the boundary of $\partial \Omega$, or at the unique critical point $C \in \Omega$ for $\alpha \in[1,2]$. For $\alpha>1$, the second alternative had been rejected by Philippin [7]. The purpose of this section is to show even $\alpha=1$ the second alternative can also be rejected unless $P^{\alpha}(x)$ is a constant in $\Omega$. This can be achieved as a consequence of the following:

Theorem 2.2. Let $u \in C^{3}(\bar{\Omega})$ is a solution to (1.1)-(1.2), if

$$
P(x)=2-2 v^{-\frac{1}{2}}-2 H u
$$

attains its minimum at the unique critical point $C \in \Omega$, then $P(x)$ is a constant on $\bar{\Omega}$.

For the proof of the Theorem 2.2, we use the strong unique continuation of analytic function, so our program is to show all order derivatives of $P(x)$ 
are vanishing at $C \in \Omega$. To this end, we choose the origin of the coordinate axes at the critical point $C \in \Omega$, then

$$
u_{1}(C)=u_{2}(C)=0,
$$

and orient the axes $x_{1}$ and $x_{2}$ in such a way that

$$
u_{12}(C)=0 .
$$

From Chen, Huang [2] and Sakaguchi [10], we know

$$
u_{11}(C)>0, \quad u_{22}(C)>0,
$$

which will be used essential in the following proof.

Proof of Theorem 2.2. Our proof is divided four steps.

Step 1: We show the derivatives of $P(x)$ up to order 2 are vanishing at $C$.

First we compute the first derivaties of $P(x)$ at $C \in \Omega$. Since at any point $x \in \Omega$

$$
\begin{aligned}
& P_{1}=v^{-\frac{3}{2}} v_{1}-2 H u_{1}=2 v^{-\frac{3}{2}} u_{i} u_{i 1}-2 H u_{1}, \\
& P_{2}=v^{-\frac{3}{2}} v_{2}-2 H u_{2}=2 v^{-\frac{3}{2}} u_{i} u_{i 2}-2 H u_{2},
\end{aligned}
$$

then from (2.3), we have

$$
P_{1}(C)=P_{2}(C)=0 .
$$

Now we compute the second derivatives of $P(x)$ at $C$. From (2.3)-(2.6), we have at $C$

$$
\begin{aligned}
P_{11} & =-\frac{3}{2} v^{-\frac{5}{2}} v_{1}^{2}+v^{-\frac{3}{2}} v_{11}-2 H u_{11}=2 u_{11}^{2}-2 H u_{11} \\
P_{12} & =-\frac{3}{2} v^{-\frac{5}{2}} v_{1} v_{2}+v^{-\frac{3}{2}} v_{12}-2 H u_{12}=0 \\
P_{22} & =-\frac{3}{2} v^{-\frac{5}{2}} v_{2}^{2}+v^{-\frac{3}{2}} v_{22}-2 H u_{22}=2 u_{22}^{2}-2 H u_{22} .
\end{aligned}
$$

Use the fact that $P(x)$ attains its minimum at $C$, we have

$$
P_{11}(C) P_{22}(C)-P_{12}^{2}(C) \geq 0 .
$$

From $(2.5),(2.9)$ and (2.11) we know

$$
\begin{aligned}
& u_{11}(C)=u_{22}(C)=H \\
& P_{11}(C)=P_{22}(C)=0 .
\end{aligned}
$$

Now we will use the induction to show that all order derivatives of $P(x)$ at $C$ are vanishing.

Step 2: As a first step for induction, we will show the derivatives of $P(x)$ of order 3,4 at $C$ are vanishing. 
First we claim

$$
\frac{\partial^{3} P}{\partial x_{1}^{k} \partial x_{2}^{3-k}}(C)=0, \quad k=0,1,2,3 .
$$

Using (2.9)-(2.11), (2.4) and (2.13) we have

$$
\begin{aligned}
P_{x_{1}^{3}}(C) & =4 H u_{x_{1}}^{3}(C) \\
P_{x_{1}^{2} x_{2}}(C) & =4 H u_{x_{1}^{2} x_{2}}(C) \\
P_{x_{1} x_{2}^{2}}(C) & =4 H u_{x_{1} x_{2}^{2}}(C) \\
P_{x_{2}^{3}}(C) & =4 H u_{x_{2}^{3}}(C) .
\end{aligned}
$$

Now, by differentiating (1.1), we obtain

$$
\begin{aligned}
u_{x_{1}^{3}}(C) & =-u_{x_{1} x_{2}^{2}}(C) \\
u_{x_{1}^{2} x_{2}}(C) & =-u_{x_{2}^{3}}(C) .
\end{aligned}
$$

To this end, use (2.8), (2.10), (2.14) and (2.20)-(2.21), we expand the function $P(x)$ in a Taylor series in a neighborhood of $C$ :

$$
\begin{aligned}
P\left(x_{1}, x_{2}\right)-P(C)= & \frac{r^{3}}{3 !}\left\{\frac{\partial^{3} P}{\partial x_{1}^{3}}(C) \times\left[\cos ^{3} \varphi-3 \cos \varphi \sin ^{2} \varphi\right]\right. \\
& \left.+\frac{\partial^{3} P}{\partial x_{1}^{2} \partial x_{2}}(C) \times\left[3 \cos ^{2} \varphi \sin \varphi-\sin ^{3} \varphi\right]\right\}+O\left(r^{4}\right),
\end{aligned}
$$

where $(r, \varphi)$ are polar coordinates: $x_{1}=r \cos \varphi, \quad x_{2}=r \sin \varphi$. Suppose

$$
\sqrt{P_{x_{1}^{3}}^{2}(C)+P_{x_{1}^{2} x_{2}}^{2}(C)} \neq 0
$$

then $P(x)$ is not a constant, so we are lead to the following representation of $P(x)$ in a neighborhood of the point $C$ :

$$
P(x)-P(C)=A_{3} \cos \left[3 \varphi-\beta_{3}\right] r^{3}+O\left(r^{4}\right),
$$

with

$$
\begin{aligned}
A_{3} & =\frac{\sqrt{P_{x_{1}^{3}}^{2}(C)+P_{x_{1}^{2} x_{2}}^{2}(C)}}{3 !}, \\
\cos \beta_{3} & =\frac{P_{x_{1}^{3}}(C)}{\sqrt{P_{x_{1}^{3}}^{2}(C)+P_{x_{1}^{2} x_{2}}^{2}(C)}},
\end{aligned}
$$

and

$$
\sin \beta_{3}=\frac{P_{x_{1}^{2} x_{2}}(C)}{\sqrt{P_{x_{1}^{3}}^{2}(C)+P_{x_{1}^{2} x_{2}}^{2}(C)}} .
$$


From (2.23) we conclude that $P(x)$ has at least 3 nodal lines forming equal angles at the point $C$, using Lemma 2.1 we know that $P(x)$ attains its minimum only on $\partial \Omega$ or at the critical point $C$, which is a contradiction. Thus $A_{3}(C)=0$ or

$$
\frac{\partial^{3} P}{\partial x_{1}^{k} \partial x_{2}^{3-k}}(C)=0 \quad \text { and } \quad \frac{\partial^{3} u}{\partial x_{1}^{k} \partial x_{2}^{3-k}}(C)=0, \quad k=0,1,2,3 .
$$

Use the similar argument we can show

$$
\frac{\partial^{4} P}{\partial x_{1}^{k} \partial x_{2}^{4-k}}(C)=0, \quad k=0,1,2,3,4
$$

and

$$
\begin{aligned}
u_{x_{1}^{4}}(C) & =u_{x_{2}^{4}}(C)=3 H^{3} \\
u_{x_{1}^{2} x_{2}^{2}}(C) & =H^{3} \\
u_{x_{1}^{3} x_{2}}(C) & =u_{x_{1} x_{2}^{3}}(C)=0 .
\end{aligned}
$$

Step 3: Now we assume all order derivatives of $P(x)$ up to $n$ are vanishing at $C$, where $n \geq 5$. Use similar argument as in Step 2 we have the following relations.

If $n=2 l, \quad l \geq 3$. Then

$$
\begin{aligned}
& u_{x_{1}^{m} x_{2}^{k-m}}(C)=u_{x_{1}^{k-m} x_{2}^{m}}(C) \\
& \text { for any } m=0,1,2, \ldots, k, \text { if } \quad k=5,6, \ldots, 2 l, \\
& u_{x_{1}^{m} x_{2}^{k-m}}(C)=0 \\
& \text { for any } m=0,1,2, \ldots, k, \quad \text { if } \quad k=5,7,9, \ldots, 2 l-1,
\end{aligned}
$$$$
u_{x_{1}^{m} x_{2}^{2 p-m}}(C)=0
$$$$
\text { for any } m=1,3,5, \ldots, 2 p-1 \text {, if } \quad p=3,4,5, \ldots, l \text {, }
$$$$
u_{x_{1}^{2 p}}(C)=u_{x_{2}^{2 p}}(C)=(2 p-1)[(2 p-3)(2 p-5) \ldots 1]^{2} H^{2 p-1}
$$$$
\text { for any } p=3,4, \ldots, l \text {. }
$$

When $l$ is even, we obtain for any $p=4,6, \ldots l$

$$
\begin{array}{r}
u_{x_{1}^{2 p}}(C) \div u_{x_{1}^{2 p-2} x_{2}^{2}}(C)=(2 p-1) \div 1 \\
u_{x_{1}^{2 p-2} x_{2}^{2}}(C) \div u_{x_{1}^{2 p-4} x_{2}^{4}}(C)=(2 p-3) \div 3
\end{array}
$$

and for any $p=3,5,7, \ldots l-1$, we have

$$
u_{x_{1}^{2 p}}(C) \div u_{x_{1}^{2 p-2} x_{2}^{2}}(C)=(2 p-1) \div 1
$$


$(2.37)$

$$
\begin{gathered}
u_{x_{1}^{2 p-2} x_{2}^{2}}(C) \div u_{x_{1}^{2 p-4} x_{2}^{4}}(C)=(2 p-3) \div 3 \\
\vdots \\
u_{x_{1}^{p+3} x_{2}^{p-3}}(C) \div u_{x_{1}^{p+1} x_{2}^{p-1}}(C)=(p+2) \div(p-2) .
\end{gathered}
$$

When $l$ is odd, we have the similar relations (2.36)-(2.38).

If $n=2 l+1, \quad l \geq 2$, a similar argument show (2.29)-(2.38) and

$$
u_{x_{1}^{m} x_{2}^{2 l+1-m}}(C)=0, \quad \text { for any } m=0,1,2, \ldots, 2 l+1
$$

hold.

Step 4: Now we show the derivatives of $P(x)$ of order $n+1$ are vanishing at $C$. We divided it two parts according to whether $n$ is odd or even.

Part A: If $n=2 l+1, \quad l \geq 2$, so $n+1=2(l+1)$ is even, we first look for the relations among $P_{x_{1}^{m} x_{2}^{n+1-m}}(C)$, where $\quad m=0,2,4, \ldots n+1$. Through calculating, we have

$$
P_{x_{1}^{n-1} x_{2}^{2}}(C)=2 n H\left\{u_{x_{1}^{n-1} x_{2}^{2}}(C)-[(2 l-1)(2 l-3) \ldots 1]^{2} H^{2 l+1}\right\} .
$$

Now, by differentiating (1.1), we obtain

$$
\frac{\partial}{\partial x_{1}^{n-1}}\left(\Delta u-u_{i} u_{j} u_{i j} v^{-1}\right)(C)=\frac{\partial}{\partial x_{1}^{n-1}}\left(2 H v^{\frac{1}{2}}\right)(C),
$$

and using the values of derivatives of $u$ up to order $n$ at $C$, this lead to

$$
u_{x_{1}^{n+1}}(C)+u_{x_{1}^{n-1} x_{2}^{2}}(C)=(n+1)[(2 l-1)(2 l-3) \ldots 1]^{2} H^{2 l+1} .
$$

From (2.40)-(2.41) and (2.43) we obtain

$$
P_{x_{1}^{n+1}}(C)=-P_{x_{1}^{n-1} x_{2}^{2}}(C) .
$$

A similar argument, it follows that

$$
P_{x_{1}^{n-1} x_{2}^{2}}(C)=-P_{x_{1}^{n-3} x_{2}^{4}}(C)=\ldots=(-1)^{l} P_{x_{2}^{n+1}}(C) .
$$

Now we will find the similar relations (2.44)-(2.45) among

$$
P_{x_{1}^{m} x_{2}^{n+1-m}}(C), \quad \text { where } \quad m=1,3,5, \ldots n .
$$

Using the same argument, we have

(2.46) $\quad P_{x_{1}^{n+1-m} x_{2}^{m}}(C)=2 n H u_{x_{1}^{n+1-m} x_{2}^{m}}(C)$, where $m=1,3,5, \ldots, n$.

Now, by differentiating (1.1), we obtain as in (2.43) the following relations

$$
u_{x_{1}^{n+1-m} x_{2}^{m}}(C)=-u_{x_{1}^{n-1-m} x_{2}^{m+2}}(C), \quad \text { for } m=1,3,5, \ldots, n-2 .
$$


From (2.46)-(2.47), it follows that

$$
P_{x_{1}^{n} x_{2}}(C)=-P_{x_{1}^{n-2} x_{2}^{3}}(C)=\cdots=(-1)^{l} P_{x_{1} x_{2}^{n}}(C) .
$$

Up to now we are able to show the derivatives of $P(x)$ of order $n+1$ are vanishing at $C$ as Step 2. Using the induction assumption, (2.44)-(2.45) and (2.48), we expand $P(x)$ in a Tayor's series in a neighborhood of the point $C$ :

$$
\begin{aligned}
P(x)-P(C) & \\
= & \frac{r^{n+1}}{(n+1) !}\left\{P_{x_{1}^{n+1}}(C) \times\left[\left(\begin{array}{c}
n+1 \\
0
\end{array}\right) \cos ^{n+1} \varphi\right.\right. \\
& \left.-\left(\begin{array}{c}
n+1 \\
2
\end{array}\right) \cos ^{n-1} \varphi \sin ^{2} \varphi+\cdots+(-1)^{l+1}\left(\begin{array}{c}
n+1 \\
n+1
\end{array}\right) \sin ^{n+1} \varphi\right] \\
& +P_{x_{1}^{n-1} x_{2}}(C) \times\left[\left(\begin{array}{c}
n+1 \\
1
\end{array}\right) \cos ^{n} \varphi \sin \varphi-\left(\begin{array}{c}
n+1 \\
3
\end{array}\right) \cos ^{n-1} \varphi \sin ^{3} \varphi\right. \\
& \left.\left.+\cdots+(-1)^{l}\left(\begin{array}{c}
n+1 \\
n
\end{array}\right) \cos \varphi \sin ^{n} \varphi\right]\right\}+O\left(r^{n+2}\right) .
\end{aligned}
$$

As in Step 2, we can show the derivatives of $P(x)$ of order $n+1$ are vanishing at $C$.

Part B: If $n=2 l, \quad l \geq 3$, a similar argument as in Part A, we have

$$
P_{x_{1}^{n+1-m} x_{2}^{m}}(C)=2 n H u_{x_{1}^{n+1-m} x_{2}^{m}}(C)
$$

for $m=0,1,2,3, \ldots, n+1$. The same analysis as in Part A leads to imply the derivatives of $P(x)$ of order $n+1$ are vanishing at $C$.

According to the unique continuation of analytic function, we know if the function $P(x)$ attains its minimum at $C$, then it must be a constant, this establishes Theorem 2.2.

Combination of Theorem 2.2 and Lemma 2.1 implies Theorem 2.

\section{The proof of Theorem 1 .}

From Section 2, we know if $u \in C^{3}(\bar{\Omega})$ is a solution to Equations (1.1)-(1.2), then the function

$$
P(x)=2-2\left(1+|D u|^{2}\right)^{-\frac{1}{2}}-2 H u
$$

attains its maximum [7] and minimum on $\partial \Omega$ unless $P(x)$ is a constant in $\bar{\Omega}$. As an application of these maximum and minimum principle of the function $P(x)$, in this section we get the size estimates of capillary free surface without gravity to complete the proof Theorem 1 and a Corollary. The proof of Theorem 1 will be divided two parts to show the different applications for maximum (minimum) principle. 
Proof of Theorem 1. Part A: Use the fact that the function $P(x)$ attains its maximum on $\partial \Omega$, we first prove (1.3)-(1.4).

Assume $P(x)$ attains its maximum at $x_{o} \in \partial \Omega$. We must have at $x_{o}$ :

$$
\begin{aligned}
\frac{1}{2} P_{s} & =\frac{1}{2}(-2)\left(-\frac{1}{2}\right) v^{-\frac{3}{2}}\left(u_{n}^{2}+u_{s}^{2}+1\right)_{s}-H u_{s} \\
& =v^{-\frac{3}{2}}\left(u_{n} u_{n s}+u_{s} u_{s s}\right)-H u_{s}=0,
\end{aligned}
$$

from boundary condition (1.2), we have

$$
\sin ^{2} \theta_{o} u_{n}^{2}=\cos ^{2} \theta_{o}+\cos ^{2} \theta_{o} u_{s}^{2},
$$

it follows that

$$
u_{n} u_{n s}=\cot ^{2} \theta_{o} u_{s} u_{s s},
$$

we conclude from (3.2) and (3.1) that

$$
\frac{1}{2} P_{s}=v^{-\frac{3}{2}}\left(\cot ^{2} \theta_{o} u_{s} u_{s s}+u_{s} u_{s s}\right)-H u_{s}=u_{s}\left[\frac{u_{s s}}{\sin ^{2} \theta_{o} v^{\frac{3}{2}}}-H\right]=0 .
$$

According to Hopf maximum principle [9], we also have at $x_{o}$ :

$$
\frac{1}{2} P_{n}=v^{-\frac{3}{2}}\left[u_{n} u_{n n}+u_{s} u_{s n}\right]-H u_{n}>0,
$$

unless $P(x)$ is a constant on $\bar{\Omega}$.

If $u_{s}\left(x_{o}\right) \neq 0$, then from $(3.3)$

$$
u_{s s}\left(x_{o}\right)=H \sin ^{2} \theta_{o} v^{\frac{3}{2}}\left(x_{o}\right) .
$$

Now we shall use (1.2), (1.9), (1.10), (3.4) and (3.5) to lead

$$
-k u_{n}|D u|^{2}>0, \quad \text { at } x_{o},
$$

which is contradiction to the strictly convexity of $\partial \Omega$. The proof of (3.6) is a long calculation. Using (3.5), at $x_{o}$, we shall rewrite (1.10) as

$u_{n n}\left(1+u_{s}^{2}\right)=2 H v^{\frac{3}{2}}+u_{s s} u_{s}^{2}+2 \cot ^{2} \theta_{o} u_{s}^{2} u_{s s}-k u_{n} u_{s}^{2}-\left(u_{s s}+k u_{n}\right)\left(1+u_{n}^{2}+u_{s}^{2}\right)$.

Using (1.9) and (3.4) we have at $x_{o}$

$$
u_{n} u_{n n}+u_{s}\left(u_{n s}-k u_{s}\right)-H u_{n} v^{\frac{3}{2}}>0,
$$

thus

(3.8) $u_{n}^{2}\left(1+u_{s}^{2}\right) u_{n n}+u_{n} u_{s}\left(1+u_{s}^{2}\right)\left(u_{n s}-k u_{s}\right)-H u_{n}^{2}\left(1+u_{s}^{2}\right) v^{\frac{3}{2}}>0, \quad$ at $\quad x_{o}$.

Combining (3.7) with (3.8) yields at $x_{o}$

$$
\begin{aligned}
& u_{n}^{2}\left[2 H v^{\frac{3}{2}}+u_{s s} u_{s}^{2}+2 \cot ^{2} \theta_{o} u_{s}^{2} u_{s s}-k u_{n} u_{s}^{2}-u_{s s}\left(1+u_{n}^{2}+u_{s}^{2}\right)-k u_{n} v\right] \\
& \quad+u_{n} u_{s}\left(1+u_{s}^{2}\right) u_{n s}-k u_{n} u_{s}^{2}\left(1+u_{s}^{2}\right)-H u_{n}^{2}\left(1+u_{s}^{2}\right) v^{\frac{3}{2}}>0 .
\end{aligned}
$$


From (3.2), (3.9) can be rewritten as

$$
\begin{aligned}
& {\left[H u_{n}^{2} v^{\frac{3}{2}}+u_{s s}\left(\cot ^{2} \theta_{o} u_{s}^{2} u_{n}^{2}-u_{n}^{2}-u_{n}^{4}\right)\right]} \\
& +\left[u_{s s} u_{s}^{2} \cot ^{2} \theta_{o}\left(1+u_{n}^{2}+u_{s}^{2}\right)-H u_{n}^{2} u_{s}^{2} v^{\frac{3}{2}}\right] \\
& -k u_{n}\left[u_{n}^{2} u_{s}^{2}+u_{n}^{2} v+u_{s}^{2}+u_{s}^{4}\right]>0 .
\end{aligned}
$$

Now we calculate (3.10), from (1.2) and (3.5), it follows that at $x_{o}$

$$
\begin{aligned}
& H u_{n}^{2} v^{\frac{3}{2}}+u_{s s} u_{n}^{2}\left(\cot ^{2} \theta_{o} u_{s}^{2}-1-u_{n}^{2}\right) \\
& =H u_{n}^{2} v^{\frac{3}{2}}-H \sin ^{2} \theta_{o} v^{\frac{3}{2}} u_{n}^{2} \sin ^{2} \theta_{o}=0,
\end{aligned}
$$

and

$$
\begin{aligned}
& u_{s s} u_{s}^{2} \cot ^{2} \theta_{o}\left(1+u_{n}^{2}+u_{s}^{2}\right)-H u_{n}^{2} u_{s}^{2} v^{\frac{3}{2}} \\
& =H \sin ^{2} \theta_{o} v^{\frac{3}{2}} \cot ^{2} \theta_{o} u_{s}^{2} v-H u_{n}^{2} u_{s}^{2} v^{\frac{3}{2}} \\
& =H u_{s}^{2} v^{\frac{3}{2}}\left(\cos ^{2} \theta_{o} v-u_{n}^{2}\right)=0,
\end{aligned}
$$

similarly we have

$$
-k u_{n}\left(u_{n}^{2} u_{s}^{2}+u_{s}^{2}+u_{s}^{4}+u_{n}^{2} v\right)=-k u_{n}\left(u_{s}^{2} v+u_{n}^{2} v\right)=-k u_{n}|D u|^{2} v
$$

Insertion (3.11)-(3.13) into (3.10) yields

$$
-k u_{n}|D u|^{2} v>0, \quad \text { at } \quad x_{o},
$$

now we complete the proof (3.6).

Thus we must have $u_{s}\left(x_{o}\right)=0$, from the expression for $P(x), x_{o}$ must be a point $A$ where $u$ attains its minimum on $\partial \Omega$ and we may use the fact that $P_{s s}(A) \leq 0$ also. It follows that

$$
0 \leq u_{s s}(A) \leq H \sin ^{2} \theta_{o} v^{\frac{3}{2}}(A) .
$$

Using the similar calculation to get (3.6), we conclude from (1.10), (1.2), (1.9) and (3.4) that

$$
k(A) \cos \theta_{o}<H-u_{s s}(A) .
$$

Insert (3.14) into (3.15) to find

$$
k(A)<\frac{H}{\cos \theta_{o}} .
$$

Moreover from the maximum principle we have $P(A)>P(C)$, it yields

$$
u(A)-u(C)<\frac{1-\sin \theta_{o}}{H} .
$$

If $P(x)$ is a constant on $\bar{\Omega}$ then a similar argument as (3.15) we have

$$
k(x) \equiv \frac{H}{\cos \theta_{o}}, \quad \text { for any } \quad x \in \partial \Omega,
$$




$$
u(x)-u(C) \equiv \frac{1-\sin \theta_{o}}{H}, \quad \text { for any } \quad x \in \partial \Omega .
$$

Which imply if $P(x)$ is a constant on $\bar{\Omega}$ then $\Omega$ is a disk with radius $\frac{\cos \theta_{o}}{H}$. Until now we complete the proof of (1.3)-(1.4).

Conversely, if at $A \in \partial \Omega$ we have $u(A)-u(C)=\frac{1-\sin \theta_{o}}{H}$ or $k(A)=$ $\frac{H}{\cos \theta_{o}}$, from strong maximum principle it follows that $P(x)$ must be a constant on $\bar{\Omega}$, so (1.7)-(1.8) hold.

Part B: Similar to Part A, use the fact that $P(x)$ attains its minimum on $\partial \Omega$, we will prove (1.5)-(1.6).

From the boundary condition (1.2), we can see that the minimum of $P(x)$ on $\partial \Omega$ must be a point $B \in \partial \Omega$ where $u$ itself is a maximum. It follows that

$$
u_{s}(B)=0, \quad u_{s s}(B) \leq 0 .
$$

A similar argument as in Part A, we have

$$
\begin{aligned}
u(B)-u(C) & \geq \frac{1-\sin \theta_{o}}{H}, \\
k(B) & \geq \frac{H}{\cos \theta_{o}},
\end{aligned}
$$

this is (1.5)-(1.6).

Conversely if $u(B)-u(C)=\frac{1-\sin \theta_{o}}{H}$ or $k(B)=\frac{H}{\cos \theta_{o}}$, then from strong maximum and Theorem $2.2 P(x)$ must be a constant on $\bar{\Omega}$, as in Part A (1.7)-(1.8) holds and $\Omega$ is a disk with radius $\frac{\cos \theta_{o}}{H}$.

When $\Omega$ is a disk of radius $\frac{\cos \theta_{o}}{H},(1.7)-(1.8)$ hold obviousily. Thus we have proved Theorem 1 .

As an another application of the minimum principle of Section 2, we prove the following Corollary for the capillary free surface area $S$ defined as

$$
S=\int_{\Omega} \sqrt{1+|D u|^{2}} d x .
$$

Corollary. Let $A$ and $B$ as in Theorem $1, V=\int_{\Omega} u d x$ is the volume of a liquid in a vertical tube, then $S$ satisfies the inequalities:

$$
\left[\sin \theta_{o}+3 H u(A)\right]|\Omega|-3 H V \leq S \leq\left[\sin \theta_{o}+3 H u(B)\right]|\Omega|-3 H V .
$$

Proof. From the fact that $P(x)$ attains its maximum at $A \in \partial \Omega$. We must have

$$
P(x) \leq P(A) \quad \text { for any } \quad x \in \Omega .
$$


So we are actually have

$$
\frac{1}{\sqrt{1+|D u|^{2}}} \geq \sin \theta_{o}+H[u(A)-u] .
$$

Since

$$
\frac{1}{\sqrt{1+|D u|^{2}}}=\left(1+|D u|^{2}\right)^{\frac{1}{2}}-\frac{|D u|^{2}}{\sqrt{1+|D u|^{2}}},
$$

we obtain from $(3.22)$

$$
H[u(A)-u]+\sin \theta_{o} \leq \sqrt{1+|D u|^{2}}-\frac{|D u|^{2}}{\sqrt{1+|D u|^{2}}} .
$$

Using the fact that $\frac{|D u|^{2}}{\sqrt{1+|D u|^{2}}}=\frac{u_{i} u_{i}}{\sqrt{1+|D u|^{2}}}$ and the divergence theorem in conjuction with (1.1)-(1.2), we find from (3.23) after an intergration over $\Omega$ that

$$
S \geq\left[3 H u(A)+\sin \theta_{o}\right]|\Omega|-3 H V .
$$

Similar using the fact that $P(x)$ attains its minimum at $B \in \partial \Omega$, we know that

$$
S \leq\left[3 H u(B)+\sin \theta_{o}\right]|\Omega|-3 H V .
$$

Inequalities (3.24)-(3.25) are optimal in the sense that the equality signs in (3.24)-(3.25) holds if and only if $\Omega$ is a disk with radius $\frac{\cos \theta_{o}}{H}$. This establishes the Corollary.

Acknowledgement. The author thanks to Prof. Shen Chun-Li and Prof. Zhou Qing for many stimulating discussions.

\section{References}

[1] J.-T. Chen, Uniqueness of minimal point and its location of capillary free surfaces over convex domain, Societe Mathematiques de France, Asterique, 118 (1984), 137143.

[2] J.-T. Chen and Wu-H. Huang, Convexity of capillary surfaces in the outer space, Invent. Math., 67 (1982), 253-259.

[3] R. Finn, Existence criteria for capillary free surfaces without gravity, Indiana Univ. Math. Jour., 32 (1983), 439-460.

[4] _ Equilibrium capillary surfaces, Springer, 1986.

[5] L. Nirenberg, On nonlinear partial differential equations and Holder continuity, Comm. Pure Appl. Math., 6 (1953), 103-156.

[6] L.E. Payne and G.A. Philippin, Some applications of the maximum principle in the problem of torsional creep, SIAM J. Appl. Math., 33 (1977), 446-455.

$[7]$

, Some maximum principles for nonlinear elliptic equations in divergence form with application to capillary surfaces and to surfaces of constant mean curvature, Nonlinear Analysis TMA, 3 (1979), 193-211. 
[8] G.A. Philippin, A minimum principle for the problem of torsional creep, Jour. Math. Anal. Appl., 68 (1979), 526-535.

[9] M.H. Protter and H.F. Weinberger, Maximum principles in differential equations, Prentice-Hall, 1967.

[10] S. Sakaguchi, Uniqueness of critical point of the solution to the prescribed constant mean curvature equation over convex domain in $R^{2}$, in 'Recent Topics on Nonlinear PDE IV', Kyoto, (1988), 129-151.

[11] R.P. Sperb, Maximum principle and their applications, Academic Press, 1981.

Received April 13, 1998.

East China Normal University

Shanghai

P.R. CHINA

E-mail address: xnma@math.ecnu.edu.cn 